\title{
Discovery of Very-high-energy Emission from RGB J2243+203 and Derivation of Its Redshift Upper Limit
}

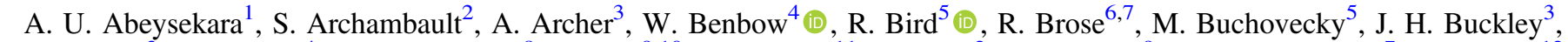
V. Bugaev ${ }^{3}$, M. Cerruti ${ }^{4}$, M. P. Connolly ${ }^{8}$, W. Cui ${ }^{9,10}$, A. Falcone ${ }^{11}$, Q. Feng ${ }^{2}$, J. P. Finley ${ }^{9}$ (i), H. Fleischhack ${ }^{7}$, L. Fortson ${ }^{12}$,

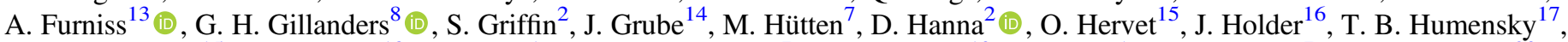
C. A. Johnson ${ }^{15}$ (D) P. Kaaret ${ }^{18}$ (D), P. Kar ${ }^{1}$, N. Kelley-Hoskins ${ }^{7}$, M. Kertzman ${ }^{19}$, D. Kieda ${ }^{1}$ (D), M. Krause ${ }^{7}$ (D), F. Krennrich ${ }^{20}$, S. Kumar ${ }^{16}$, M. J. Lang ${ }^{8}$, G. Maier ${ }^{7}$ (D), S. McArthur ${ }^{9}$, P. Moriarty ${ }^{8}$, R. Mukherjee ${ }^{21}$ (D), D. Nieto ${ }^{17}$ (D) , S. O’Brien ${ }^{22}$, R. A. Ong ${ }^{5}$, A. N. Otte ${ }^{23}$, N. Park ${ }^{24}$, A. Petrashyk ${ }^{17}$, M. Pohl ${ }^{6,7}$ (D) A. Popkow ${ }^{5}$ (D) E. Pueschel ${ }^{7}$ (D), J. Quinn ${ }^{22}$, K. Ragan ${ }^{2}$, P. T. Reynolds ${ }^{25}$, G. T. Richards ${ }^{23}$ (D) E. Roache ${ }^{4}$, C. Rulten ${ }^{12}$, I. Sadeh ${ }^{7}$, M. Santander ${ }^{21}$, G. H. Sembroski ${ }^{9}$, K. Shahinyan ${ }^{12}$ (ID) D. Staszak ${ }^{24}$, I. Telezhinsky ${ }^{6,7}$ (D) , J. Tyler ${ }^{2}$, V. V. Vassiliev ${ }^{5}$, S. P. Wakely ${ }^{24}$, O. M. Weiner ${ }^{17}$, A. Weinstein ${ }^{20}$, P. Wilcox ${ }^{18}$, A. Wilhelm ${ }^{6,7}$, D. A. Williams ${ }^{15}$ (D), and B. Zitzer ${ }^{2}$

${ }^{1}$ Department of Physics and Astronomy, University of Utah, Salt Lake City, UT 84112, USA

${ }^{2}$ Physics Department, McGill University, Montreal, QC H3A 2T8, Canada

${ }^{3}$ Department of Physics, Washington University, St. Louis, MO 63130, USA

${ }^{4}$ Fred Lawrence Whipple Observatory, Harvard-Smithsonian Center for Astrophysics, Amado, AZ 85645, USA

${ }_{6}^{5}$ Department of Physics and Astronomy, University of California, Los Angeles, CA 90095, USA

${ }^{6}$ Institute of Physics and Astronomy, University of Potsdam, D-14476 Potsdam-Golm, Germany DESY, Platanenallee 6, D-15738 Zeuthen, Germany

${ }_{9}^{8}$ School of Physics, National University of Ireland Galway, University Road, Galway, Ireland

${ }^{9}$ Department of Physics and Astronomy, Purdue University, West Lafayette, IN 47907, USA

${ }^{10}$ Department of Physics and Center for Astrophysics, Tsinghua University, Beijing 100084, China

${ }^{11}$ Department of Astronomy and Astrophysics, 525 Davey Lab, Pennsylvania State University, University Park, PA 16802, USA

${ }_{13}^{12}$ School of Physics and Astronomy, University of Minnesota, Minneapolis, MN 55455, USA

${ }^{13}$ Department of Physics, California State University-East Bay, Hayward, CA 94542, USA Department of Physics, Stevens Institute of Technology, Hoboken, NJ 07030, USA

${ }^{15}$ Santa Cruz Institute for Particle Physics and Department of Physics, University of California, Santa Cruz, CA 95064, USA

${ }^{16}$ Department of Physics and Astronomy and the Bartol Research Institute, University of Delaware, Newark, DE 19716, USA

${ }^{17}$ Physics Department, Columbia University, New York, NY 10027, USA

${ }^{18}$ Department of Physics and Astronomy, University of Iowa, Van Allen Hall, Iowa City, IA 52242, USA

${ }^{19}$ Department of Physics and Astronomy, DePauw University, Greencastle, IN 46135-0037, USA

${ }^{20}$ Department of Physics and Astronomy, Iowa State University, Ames, IA 50011, USA

${ }^{21}$ Department of Physics and Astronomy, Barnard College, Columbia University, NY 10027, USA

${ }^{22}$ School of Physics, University College Dublin, Belfield, Dublin 4, Ireland

${ }^{23}$ School of Physics and Center for Relativistic Astrophysics, Georgia Institute of Technology, 837 State Street NW, Atlanta, GA 30332-0430, USA

${ }^{24}$ Enrico Fermi Institute, University of Chicago, Chicago, IL 60637, USA

${ }^{25}$ Department of Physical Sciences, Cork Institute of Technology, Bishopstown, Cork, Ireland

Received 2017 May 18; revised 2017 September 11; accepted 2017 September 15; published 2017 November 10

\begin{abstract}
Very-high-energy (VHE; > $100 \mathrm{GeV}$ ) gamma-ray emission from the blazar RGB J2243+203 was discovered with the VERITAS Cherenkov telescope array, during the period between 2014 December 21 and 24. The VERITAS energy spectrum from this source can be fitted by a power law with a photon index of $4.6 \pm 0.5$, and a flux normalization at $0.15 \mathrm{TeV}$ of $(6.3 \pm 1.1) \times 10^{-10} \mathrm{~cm}^{-2} \mathrm{~s}^{-1} \mathrm{TeV}^{-1}$. The integrated Fermi-LAT flux from 1 to $100 \mathrm{GeV}$ during the VERITAS detection is $(4.1 \pm 0.8) \times 10^{-8} \mathrm{~cm}^{-2} \mathrm{~s}^{-1}$, which is an order of magnitude larger than the four-year-averaged flux in the same energy range reported in the 3FGL catalog, $\left(4.0 \pm 0.1 \times 10^{-9} \mathrm{~cm}^{-2} \mathrm{~s}^{-1}\right)$. The detection with VERITAS triggered observations in the X-ray band with the Swift-XRT. However, due to scheduling constraints Swift-XRT observations were performed $67 \mathrm{hr}$ after the VERITAS detection, rather than simultaneously with the VERITAS observations. The observed X-ray energy spectrum between 2 and $10 \mathrm{keV}$ can be fitted with a power law with a spectral index of $2.7 \pm 0.2$, and the integrated photon flux in the same energy band is $(3.6 \pm 0.6) \times 10^{-13} \mathrm{~cm}^{-2} \mathrm{~s}^{-1}$. EBL-model-dependent upper limits of the blazar redshift have been derived. Depending on the EBL model used, the upper limit varies in the range from $z<0.9$ to $z<1.1$.
\end{abstract}

Key words: galaxies: individual (RGB J2243+203)

\section{Introduction}

RGB J2243+203 was first reported as a radio source in the MIT-Green Bank (RGB) catalog by Griffith et al. (1990). The optical energy spectrum of the source was studied by LaurentMuehleisen et al. (1998), who found the observed energy spectrum to be featureless, leading to its classifications as a $\mathrm{BL}$ Lac type blazar. Laurent-Muehleisen et al. (1999) studied the cross-correlation of a ROSAT All-Sky Survey source list with an extensive sample of radio sources from an RGB sky survey, including RGB J2243+203, and found that the X-rayto-radio flux ratios of the RGB BL Lacertae objects are shown to peak between the low-energy-peaked BL Lacs (LBLs) and the high-energy-peaked BL Lacs (HBLs). Therefore, LaurentMuehleisen et al. (1999) categorized RGB J2243+203 as an 
intermediate-frequency-peaked BL Lac (IBL). Twelve years later, the second Fermi-LAT AGN catalog (2LAC; Ackermann et al. 2011) identified RGB J2243+203 as a BL Lac object with a synchrotron peak corresponding to an HBL. The biggest difference between these two studies is LaurentMuehleisen et al. (1999) used the collective properties of a sample of BL Lacertae objects and classified the whole sample as IBLs, but Ackermann et al. (2011) used the properties of RGB J2243+203 to classify the source as an HBL.

Meisner \& Romani (2010) attempted to measure the redshift of RGB J2243+203. Following Sbarufatti et al. (2005), Meisner \& Romani (2010) assumed BL Lac host galaxies are standard candles with $M_{R}=-22.9 \pm 0.5$, and estimated the redshift using the apparent magnitude of the host galaxy. They were unable to resolve the host galaxy of RGB J2243+203. Therefore, a lower limit on the redshift, $z>0.39$, was calculated using the upper limit of the host galaxy flux. However, this method is susceptible to the accuracy of the assumptions. Section 3 of this paper estimates upper limits for the redshift of the source using the method proposed by Georganopoulos et al. (2010).

The VERITAS discovery of $\mathrm{TeV}$ gamma-ray emission from RGB J2243+203 was announced on 2014 December 24 in Astronomer's Telegram \#6849. Section 2.1 describes the details of the VERITAS results, Section 2.2 describes the analysis of simultaneous and historical Fermi-LAT data, and Section 2.3 describes the measurements of contemporaneous and historical Swift-XRT data. Section 3 derives EBL-model-dependent redshift upper limits, and Section 4 features a discussion.

\section{Observations and Data Analysis}

\subsection{VERITAS Observations}

VERITAS is an array of four imaging atmospheric Cherenkov telescopes (IACTs) that follow the Davies-Cotton design, with $12 \mathrm{~m}$ diameter reflectors (Holder et al. 2008). The array is located at the Fred Lawrence Whipple Observatory in southern Arizona $\left(31^{\circ} 40^{\prime} \mathrm{N}, 110^{\circ} 57^{\prime} \mathrm{W}, 1.3 \mathrm{~km}\right.$ a.s.l.), and is sensitive to gamma-rays in the energy range from $\sim 85 \mathrm{GeV}$ to $\sim 30 \mathrm{TeV}$. It has the sensitivity to detect a point source at five standard deviations (at $5 \sigma$ ) with a brightness of $1 \%$ of the Crab Nebula flux with an exposure of $<25 \mathrm{hr}$. The energy of gammarays can be measured with a resolution of $15 \%-25 \%$ and the angular resolution is better than 0.1 at $1 \mathrm{TeV}$.

On 2014 December 21, an automated Fermi-LAT analysis pipeline used by VERITAS collaborators indicated that the daily Fermi-LAT flux of RGB J2243+203 was marginally elevated from the average flux. VERITAS observed the source from 2014 December 21 (Modified Julian Date (MJD) 57012) through 2014 December 24 (MJD 57015) for a total of 264 minutes of good-quality data. Within this time interval, the source was observed from when the night began until the elevation of the source went below $40^{\circ}$. The average elevation of the observations is $53^{\circ}$. The data set has been analyzed with a reflected-region background model (Aharonian et al. 2001), and the minimum two-telescope triggering criterion described in Holder et al. (2006). On the first night, the source was observed for 37 minutes, during which $204 \mathrm{ON}$ events and 873 OFF events were detected with a background normalization factor $\alpha$ of 0.167 , yielding an excess of 58.5 events corresponding to a detection significance of $4.2 \sigma$. With an

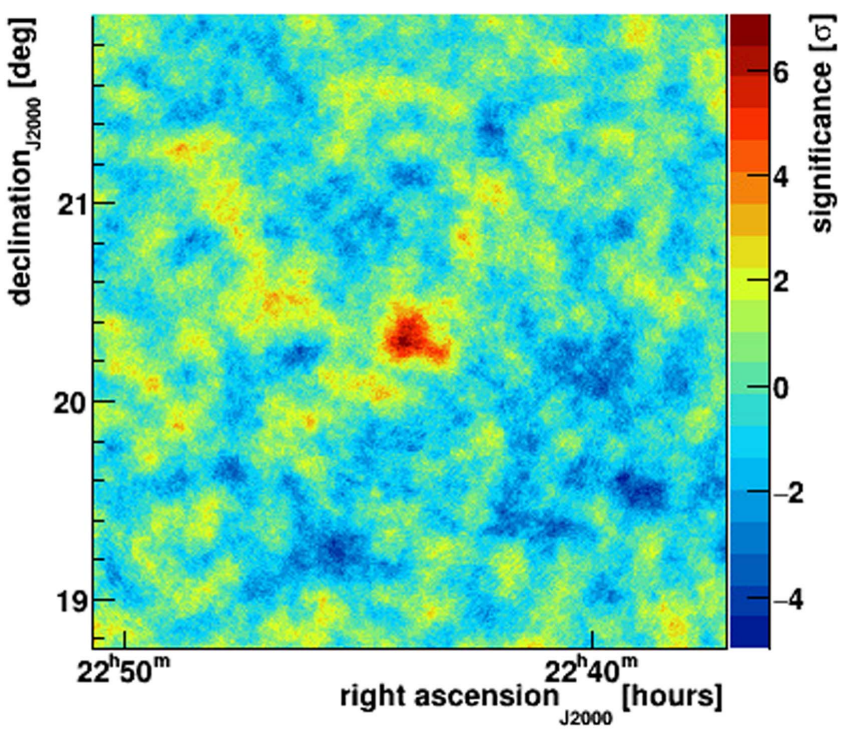

Figure 1. VERITAS skymap of the region around RGB J2243+203.

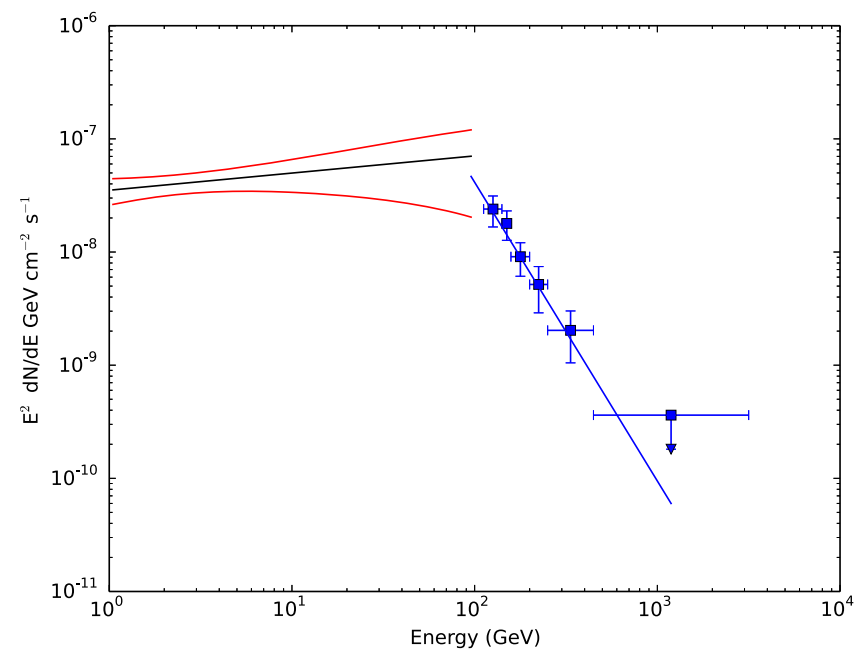

Figure 2. Gamma-ray SED of RGB J2243+203 during the flare from 2014-1221 01:44:00 UTC to 2014-12-24 03:34:00 UTC (MJD 57012.072 to MJD 57015.14861). The solid black line represents the best-fit SED of the FermiLAT detection, and the red bow tie represents the $1 \sigma$ uncertainty of the likelihood fit to the Fermi-LAT data. VERITAS fluxes are shown as blue points, and the solid blue line represents the best fit to the VERITAS data.

additional 227 minutes, VERITAS detected 1086 ON events and 5393 OFF events with a background normalization factor $\alpha$ of 0.167 , yielding an increased cumulative excess of 187 events, and the cumulative significance increased to $5.6 \sigma$. The excess is consistent with a point-like source within the VERITAS point-spread function. Observations ceased on 2014 December 24 because moonlight precluded VERITAS observations with a sufficiently low-energy threshold. The significance map of the region, centered at the source location, is shown in Figure 1. The gamma-ray energy spectrum above $112 \mathrm{GeV}$ can be fitted with a power law with a spectral index of $4.6 \pm 0.5$, and flux normalization at $0.15 \mathrm{TeV}$ of $(6.3 \pm 1.1) \times 10^{-10} \mathrm{~cm}^{-2} \mathrm{~s}^{-1} \mathrm{TeV}^{-1}$. The VERITAS gammaray energy spectrum is shown in Figure 2. The best fit has a $\chi^{2} / \mathrm{NDF}$ of $0.55 / 3$, with no significant indication of a spectral break or curvature. The integrated gamma-ray photon flux above $112 \mathrm{GeV}$ averaged over the VERITAS observations is 

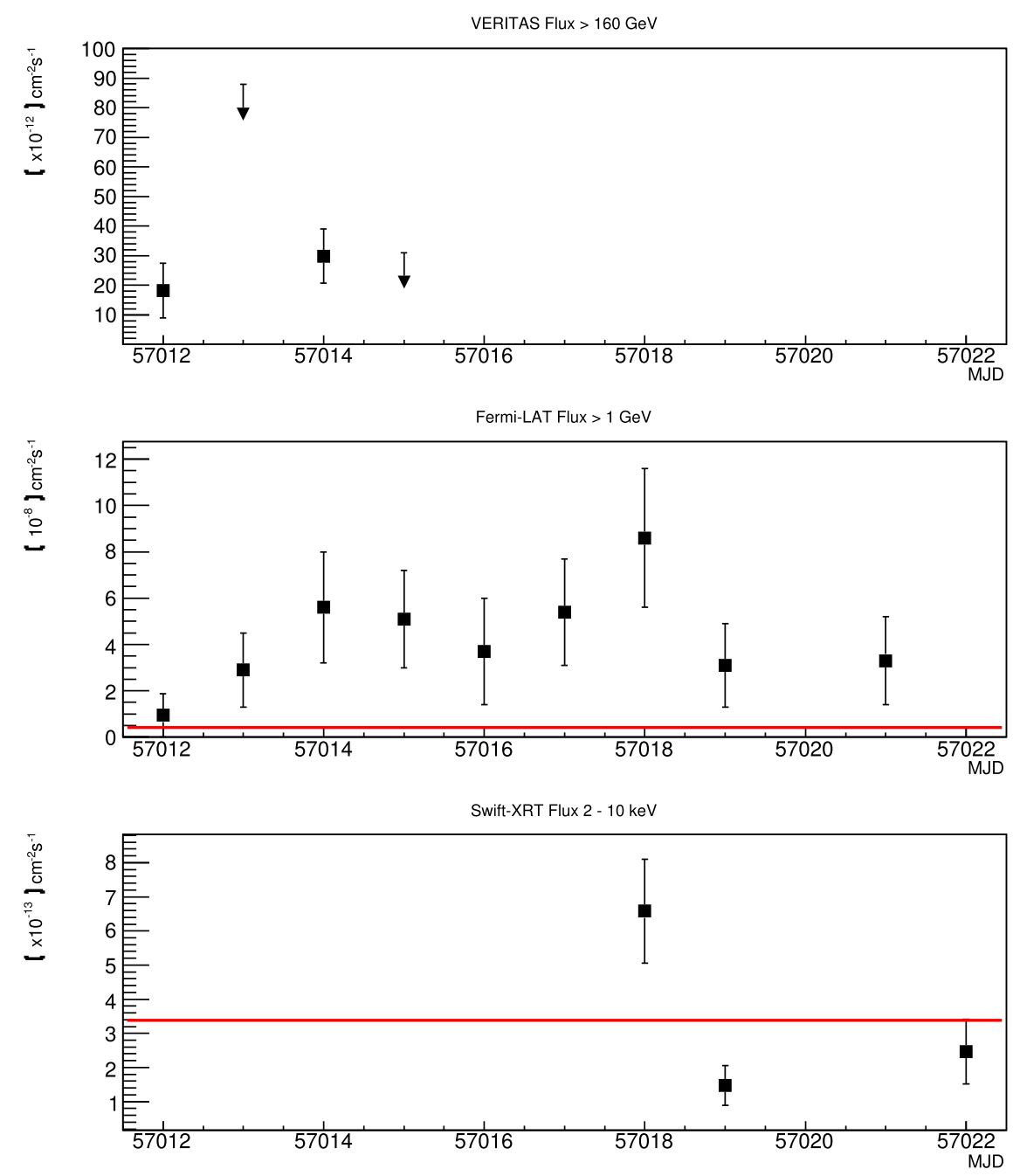

Figure 3. Top: 2014 December VERITAS light curve from the 21st (MJD 57012) to 24th (MJD 57015) in nightly bins. 95\% confidence level upper limits are shown for the bins with a significance less than 2 $\sigma$. Middle: Fermi-LAT light curve from 2014 December 21 (MJD 57012) to 31(MJD 57022) in nightly bins. The red line shows the four-year-averaged flux as reported in the 3FGL catalog. Bottom: Swift-XRT light curve. The red line shows the best fit to a constant.

$(7.8 \pm 1.4) \times 10^{-11} \mathrm{~cm}^{-2} \mathrm{~s}^{-1}$, which corresponds to $14 \%$ of the Crab Nebula flux.

The centroid of the detection was determined by fitting a symmetric two-dimensional Gaussian to the excess counts map. The best fit resulted in a centroid at R.A. $=340.97$ $\left(22^{\mathrm{h}} 43^{\mathrm{m}} 52^{\mathrm{s}}\right)$ and decl. $=+20^{\circ} .32$, with a statistical uncertainty of 0.02 and systematic uncertainty of 0.007 . This new veryhigh-energy source is cataloged as VER J2243+203. This source position is also consistent with the centroid of the RGB $\mathrm{J} 2243+203$ measured using very long baseline interferometry (VLBI), with a probability of 0.87 .

Before this observation, the blazar was observed by VERITAS between 2009 September and October for a total of about $4 \mathrm{hr}$ of good-quality data. The past observations did not show evidence for $\mathrm{TeV}$ emission from the blazar, and the upper limit on the flux above $170 \mathrm{GeV}$ at the $95 \%$ confidence level (Rolke et al. 2005) is $<2.1 \%$ of the Crab Nebula flux (Archambault et al. 2016).

The VERITAS light curve from 2014 December 21 (MJD 57012 ) to 2014 December 24 (MJD 57015) in nightly bins is shown in the top subfigure in Figure 3. On 2014 December 21 and 23, gamma-ray like events were detected with a significance of $4.2 \sigma$ in each day. No significant detection of gamma-ray like events was recorded at the source location on
2014 December 22 and 24. Therefore, 95\% upper limits are marked for those two days.

\subsection{Fermi Large Area Telescope (LAT) Observations}

The most recent Fermi-LAT source catalog, 3FGL, (Acero et al. 2015) reported the detection of sources in the energy range of $100 \mathrm{MeV}$ to $300 \mathrm{GeV}$, using the first four years of science data from Fermi-LAT. RGB J2243+203 (3FGL $\mathrm{J} 2243.9+2021$ ) was detected with a statistical significance of $45.9 \sigma$, and the average flux between $1 \mathrm{GeV}$ and $100 \mathrm{GeV}$ in the four-year data set is $(4.1 \pm 0.2) \times 10^{-9} \mathrm{~cm}^{-2} \mathrm{~s}^{-1}$. The variability of the flux is measured using the variability index defined in Abdo et al. (2010b) with an index of 59.1 for the four-year data set. The energy spectrum in the $100 \mathrm{MeV}$ to $100 \mathrm{GeV}$ energy range was described as a simple power-law energy spectrum with a spectral index of $1.79 \pm 0.03$.

The Second Fermi-LAT Catalog of High-Energy Sources, 2FHL, (Ackermann et al. 2016) also reported a detection of RGB J2243+203 at energies above $50 \mathrm{GeV}$. The source was detected with a TS $=123.5$, and the average flux between $50 \mathrm{GeV}$ and $2 \mathrm{TeV}$ is $4.09 \pm 1.24 \times 10^{-11} \mathrm{~cm}^{-2} \mathrm{~s}^{-1}$. The energy spectrum was described as a simple power-law spectrum with a spectral index of $4.16 \pm 0.97$. 


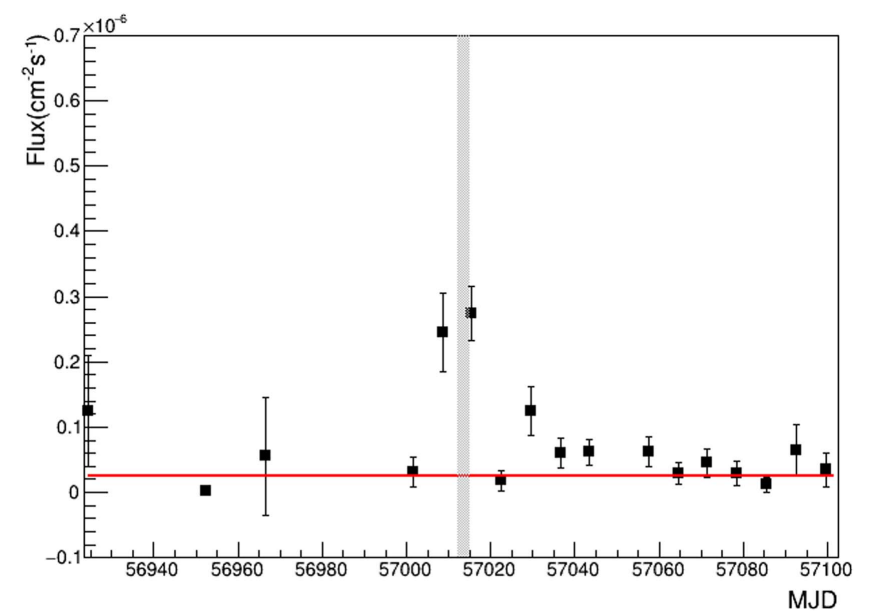

Figure 4. Fermi-LAT light curve of RGB J2243+203 with seven-day bins. The solid red line indicates the four-year, time-averaged flux reported in the Fermi-LAT 3FGL catalog. Flux measurements are not plotted for bins with TS less than 4 . The shaded vertical strip is the VERITAS observation window of the source.

In order to obtain a quasi-simultaneous $\mathrm{GeV}$ gamma-ray energy spectrum of the blazar, the Fermi-LAT data set has been analyzed from 2014 December 21 01:44:00 UTC to 2014 December 24 03:34:00 UTC (MJD 57012.072 to MJD 57015.14861), overlapping the time of the VERITAS detection of RGB J2243+203. The data set has been analyzed using the Pass 8 Fermi-LAT analysis tools, with the standard instrument response function P8R2_SOURCE_V6. The Galactic diffuse emission and the isotropic diffuse emission were modeled with gll_iem_v06.fits and iso_P8R2_SOURCE_V6_v06.txt, respectively. Photons belonging to Fermi-LAT event class 128 within a region of interest $10^{\circ} \times 10^{\circ}$ centered on the blazar position were analyzed, with the selection cuts of rocking angle $<52^{\circ}$ and zenith angle of $<90^{\circ}$. An energy cut with a minimum photon energy of $1 \mathrm{GeV}$, and maximum photon energy of $100 \mathrm{GeV}$ is applied.

This analysis yields a detection of RGB J2243+203 with a $\sqrt{\mathrm{TS}}=9.2$. The gamma-ray energy spectrum between $1 \mathrm{GeV}$ and $100 \mathrm{GeV}$ can be fitted with a power law with a spectral index of $1.8 \pm 0.2$, and a flux normalization at $2.2 \mathrm{GeV}$ of $(8.4 \pm 1.7) \times 10^{-12} \mathrm{~cm}^{-2} \mathrm{~s}^{-1} \mathrm{MeV}^{-1}$. Spectral shapes more complex than a simple power law do not result in a significant improvement in the fit. The integrated photon flux averaged over the selected time window is $(4.1 \pm 0.8) \times 10^{-8} \mathrm{~cm}^{-2} \mathrm{~s}^{-1}$, which is an order of magnitude larger than the four-yearaveraged 3FGL flux in the same energy range; $(4.0 \pm 0.1) \times 10^{-9} \mathrm{~cm}^{-2} \mathrm{~s}^{-1}$. The light curve of the integral flux between $1 \mathrm{GeV}$ and $100 \mathrm{GeV}$ with daily binning starting from MJD 57012 (start of VERITAS observations) to MJD 57022 (the end of Swift-XRT observations) is shown in the middle subfigure of Figure 3 . The red line provides the average flux reported in the 3FGL catalog. The 3FGL catalog asserted that the RGB J2243+203 GeV flux did not exhibit significant variability when averaged month by month. Figure 4 shows the 6-month light curve with a binning of 7 days. The timeaveraged flux over four years is shown in a solid red line. The highest flux recorded within this six months is over a two-week period that includes both 2014 December 17 and 24 UTC, which coincides with the VERITAS detection.
Table 1

Summary of the Swift-XRT Observations

\begin{tabular}{lcccc}
\hline \hline $\begin{array}{l}\text { Observation } \\
\text { Start }\end{array}$ & Live Time & $\begin{array}{c}\text { counts per } \\
\text { second }\end{array}$ & Spectral Index & $\begin{array}{c}\text { Integrated } \\
\text { Flux } \\
(2-10 \mathrm{keV}) \\
\times 10^{-13} \\
\text { MJD }\end{array}$ \\
& $(\mathrm{s})$ & $\times 10^{-2}$ & & $\mathrm{~cm}^{-2} \mathrm{~s}^{-1}$ \\
\hline \multicolumn{5}{c}{ Archival Observations } \\
\hline 54956.200 & 5414.137 & $29.0 \pm 0.7$ & $2.62 \pm 0.06$ & $26.4 \pm 1.5$ \\
56039.338 & 1066.342 & $6.9 \pm 0.8$ & $2.9 \pm 0.3$ & $4.3 \pm 1.2$ \\
56460.179 & 1028.892 & $2.1 \pm 0.5$ & $2.5_{-0.6}^{+0.7}$ & $3.6 \pm 2.0$ \\
56842.811 & 1997.831 & $3.9 \pm 0.4$ & $2.8 \pm 0.3$ & $2.8 \pm 0.7$ \\
\hline \multicolumn{5}{c}{ VERITAS-triggered Observations } \\
\hline 57017.945 & 1043.866 & $8.1 \pm 0.1$ & $2.7 \pm 0.3$ & $6.6 \pm 1.5$ \\
57018.944 & 1001.413 & $3.7 \pm 0.6$ & $3.2_{-0.5}^{+0.6}$ & $1.5 \pm 0.6$ \\
57019.013 & 1418.466 & $3.6 \pm 0.5$ & $\ldots$ & $\ldots$ \\
57020.205 & 1051.358 & $4.0 \pm 0.6$ & $\ldots$ & $\ldots$ \\
57021.735 & 874.052 & $4.4 \pm 0.7$ & $3.1 \pm 0.6$ & $2.5 \pm 0.9$ \\
\hline
\end{tabular}

Note. For each day the X-ray energy spectrum between 2 and $10 \mathrm{keV}$ can be fitted with a power law. The third column shows the spectral index of the best fit, and the fourth column shows the integrated flux between 2 and $10 \mathrm{keV}$. Spectral fitting failed for the observations on MJD 57019.013 and MJD 57020.205 .

\subsection{Swift X-Ray Telescope (XRT) Observations}

The VERITAS discovery of TeV emission from RGB J2243 +203 triggered pointed observations with the Swift Space Telescope (Gehrels et al. 2004). The focusing X-ray Telescope (XRT) on board Swift is sensitive to X-ray photons in the energy range from 0.3 to $10 \mathrm{keV}$. Swift observed the source five times between MJD 57017.945 (2014 December 26 22:40:48.000 UTC) and 57021.735 (2014 December 30 17:38:24.000 UTC). The Swift data archive also contains previous observations of the source taken between MJD 54956.2 and 56842.811. Photon Counting (PC) readout mode was used to carry out the observations, and there is no photon pile-up in the data. The data were calibrated and cleaned with standard criteria, with the xrtpipeline task using the calibration files as available in the Swift CALDB version 20140120. Spectral analysis was performed with events recorded within a circle of 20 pixels, corresponding to a $\sim 45^{\prime \prime}$ radius centered on the source position. A nearby circular region of 40-pixel radius was used to estimate the background. The most recent response matrices (v.014) available in the Swift CALDB were used.

Table 1 summarizes the Swift-XRT observations of RGB $\mathrm{J} 2243+203$. For the archival data and triggered observations, the X-ray energy spectrum between $2 \mathrm{keV}$ and $10 \mathrm{keV}$ was fitted with a power law. The table shows the spectral index for the best-fit energy spectrum, and the integrated flux between 2 and $10 \mathrm{keV}$. To ensure a reasonable constraint on the measured spectra, SED-fitting was performed with bins that have at least of 20 counts in each bin. However, the observations on MJD 57019.013 and MJD 5720.205 do not have enough bins with more than 20 counts to perform a spectral fit. The cumulative data set of all five VERITAS-triggered observations can also be fitted with a power law with a spectral index of $2.7 \pm 0.2$, and the integrated photon flux in the same energy band is $(3.6 \pm 0.6) \times 10^{-13} \mathrm{~cm}^{-2} \mathrm{~s}^{-1}$.

The X-ray flux for the first archival observation is larger than all the other flux measurements, including the triggered 
observations, clearly representing an X-ray-flux-elevated state. The average flux of the archival observations and the triggered observations without the first measurement is $(3.5 \pm 1.2) \times$ $10^{-13} \mathrm{~cm}^{-2} \mathrm{~s}^{-1}$. The light curve of the triggered observations is shown in the bottom subfigure of Figure 3. The red line shows the average flux. Swift-XRT observations on 2014 December 26 show a mildly elevated flux, but the following nights did not show strong X-ray activity.

\section{Redshift Upper Limit}

An upper limit on the redshift of RGB J2243+203 can be derived using the Fermi-LAT-measured energy spectrum and the VERITAS-measured energy spectrum. This method was proposed by Georganopoulos et al. (2010) and has been applied by others to derive redshift upper limits (e.g., Aleksić et al. (2011) and H.E.S.S. Collaboration et al. 2013). This method does not assume any source emission models. The only assumption is that the observed VERITAS high-energy component of the energy spectrum is softer than the extrapolation of the Fermi-LAT GeV energy spectrum. At a given energy within the VERITAS observed energy range,

$$
F(E)_{\text {obs }}<F(E)_{\text {ext }} \text {, }
$$

where $F(E)_{\text {obs }}$ is the observed VHE flux at energy $E$, and $F(E)_{\text {ext }}$ is the flux derived at energy $E$ from the extrapolation of the $\mathrm{GeV}$ energy spectrum measured quasi-simultaneous with VERITAS. Using the above assumption, Equation (1) in Georganopoulos et al. (2010) gives the upper limit to the EBL-induced pair absorption optical depth at a given energy $\tau_{\max }(E)$,

$$
\tau_{\max }(E)=\ln \left(F(E)_{\mathrm{ext}} / F(E)_{\mathrm{obs}}\right) .
$$

Similar to Aleksić et al. (2011) and H.E.S.S. Collaboration et al. (2013), this equation can be modified to include a correction term for the error on the observed VERITAS flux,

$$
\tau_{\max }(E)=\ln \left(\frac{F(E)_{\mathrm{ext}}}{F(E)_{\mathrm{obs}}-1.64 \times \Delta F(E)_{\mathrm{obs}}}\right),
$$

where $\Delta F(E)_{\text {obs }}$ accounts for the error in the VERITAS flux measurements. $\tau_{\max }(E)$ gives the upper limit of $\tau(E)$ as a function of energy. The upper limit of $\tau(E)$ can be translated into an upper limit on the redshift by comparing with EBL models. Intrinsic hardening of the energy spectrum at energies greater than the Fermi-LAT component of the energy spectrum would increase the derived upper limit. Although some hadronic models predict a spectral hardening at high energies (e.g., Cerruti et al. 2017), in general, spectral hardening at $\mathrm{TeV}$ energies is not expected. The derived redshift upper limit would be lower if there were intrinsic softening of the energy spectrum. Softening of the energy spectrum at higher energies is possible, but a lower redshift would not contradict the derived upper limit.

In Figure 5 the upper limit of $\tau$ as a function of energy $\left(\tau_{\max }(E)\right)$ is shown by a solid black line. The region marked in gray is the excluded region. The $\tau(E, z)$ distributions derived with the EBL model proposed by Gilmore et al. (2012) at redshifts of 1.2 and 1.0 are shown with dotted lines.

The minimum differences between $\tau(E, z)$ and $\tau_{\max }$ $\left(D_{\min }(z)=\tau(z, E)-\tau_{\max }(E)\right)$, over all energy bins of the

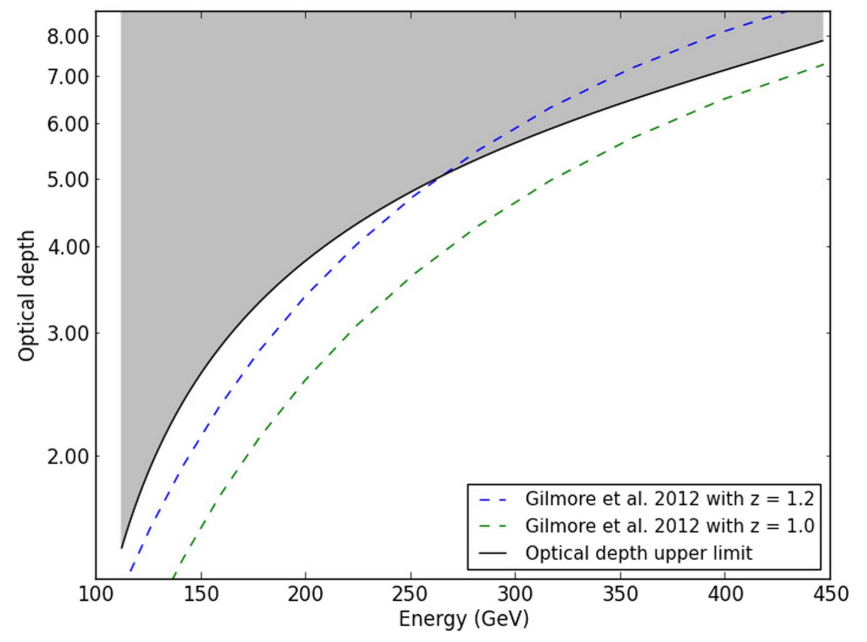

Figure 5. The black solid line indicates the maximum EBL-induced pair absorption optical depth at different energies along the line of sight to RGB $\mathrm{J} 2243+203$ that is derived from VERITAS and Fermi-LAT data. The two dashed lines indicate the modeled (Gilmore et al. 2012) EBL-induced pair absorption optical depth as a function of energy for redshifts of 1.2 and 1.0. The shaded area is the excluded region.

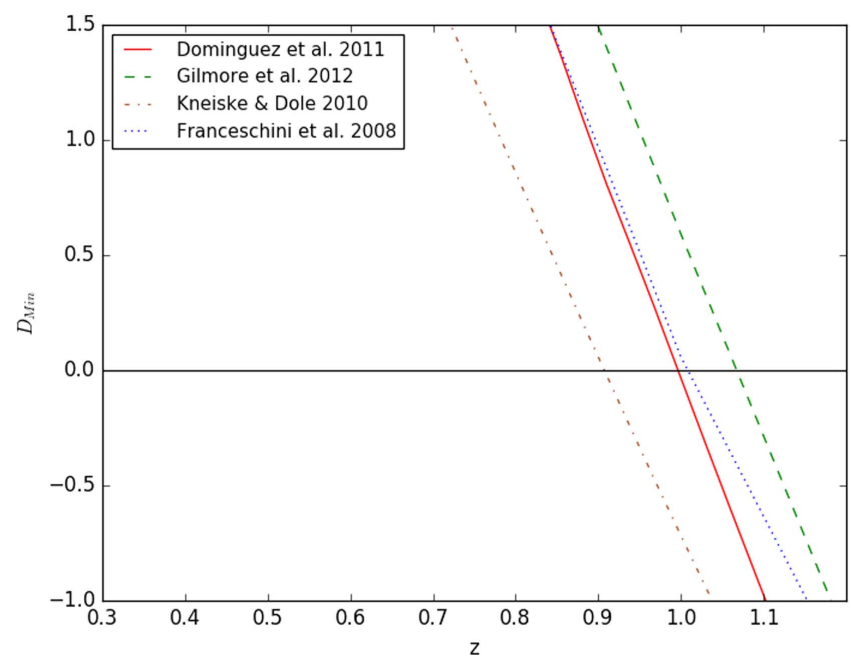

Figure 6. The minimum difference between the calculated maximum EBLinduced pair absorption optical depth and the model predicted EBL-induced pair absorption optical depth as a function of redshift.

VERITAS spectrum, for three different state-of-the-art EBL models, are shown in Figure 6.

At the $95 \%$ confidence interval upper limit of the redshift, $D_{\min }$ becomes zero. As shown in Figure 6, the Gilmore et al. (2012), Domínguez et al. (2011), Kneiske \& Dole (2010), and Franceschini et al. (2008) models predict upper limits of $z<1.1, z<1.0, z<0.9$ and $z<1.0$, respectively.

\section{Discussion}

The simultaneous gamma-ray SED with Fermi-LAT and VERITAS is shown in Figure 2. The VERITAS flux points are not corrected for EBL absorption. No other instruments observed RGB J2243+203 simultaneously with VERITAS. Observations by Swift-XRT in X-rays were triggered after the discovery of gamma-ray activity. The observed X-ray flux on 
2014 December 26 shows a marginally elevated flux, but archival data shows the following nights do not have elevated fluxes. While it is possible that the gamma-ray flare from RGB J2243+203 was not associated with a flare at longer wavelengths (i.e., an orphan gamma-ray flare), it is more likely than any activity at longer wavelengths was missed, and that the Swift-XRT observations taken $67 \mathrm{hr}$ after the VERITAS flare were measuring a post-flare state. It is well known that blazar emission models, even in the simplest one-zone synchrotron self-Compton scenario, are degenerate in the absence of simultaneous measurements of the synchrotron and inverse-Compton peaks (Tavecchio et al. 1998; Boettcher et al. 2012; Cerruti et al. 2013). For this reason, no specific modeling of the SED was performed.

\section{Conclusion}

VHE gamma-ray emission has been detected from RGB $\mathrm{J} 2243+203$ by the VERITAS observatory. Earlier VERITAS upper limits from this source provide evidence that the source is variable in the VHE band, and suggest that the detection was obtained during an active period of the source. The gamma-ray energy spectrum above $112 \mathrm{GeV}$ can be fitted with a power law with a spectral index of $4.6 \pm 0.5$, and a flux normalization at $0.15 \mathrm{TeV}$ of $(0.63 \pm 0.11) \times 10^{-9} \mathrm{~cm}^{-2} \mathrm{~s}^{-1} \mathrm{TeV}^{-1}$. The gamma-ray energy spectrum between $1 \mathrm{GeV}$ and $100 \mathrm{GeV}$ measured by Fermi-LAT quasi-simultaneous with VERITAS observations can be fitted with a power law with a spectral index of $1.8 \pm 0.2$, and a flux normalization at $2.2 \mathrm{GeV}$ of $(8.4 \pm 1.7) \times 10^{-12} \mathrm{~cm}^{-2} \mathrm{~s}^{-1} \mathrm{MeV}^{-1}$. The integrated photon flux averaged over the time window is $(4.1 \pm 0.8) \times$ $10^{-8} \mathrm{~cm}^{-2} \mathrm{~s}^{-1}$, which is an order of magnitude larger than the four-year-averaged flux in the same energy range, $(4.0 \pm 0.1) \times 10^{-9} \mathrm{~cm}^{-2} \mathrm{~s}^{-1}$. This supports the conclusion that the source was also active in the $\mathrm{GeV}$ energy band during the time of VHE detection. The VERITAS discovery of $\mathrm{TeV}$ emission from RGB J2243+203 triggered observations with the Swift-XRT. However, the first Swift-XRT observation was obtained $67 \mathrm{hr}$ after the VERITAS observations. This measurement indicates a mildly elevated state in flux, but the following nights have the same level of flux as archival data. While Meisner \& Romani (2010) placed a lower limit for the redshift of $z>0.39$, this article places EBL-model-dependent upper limits for the redshift, in the range from $z<0.9$ to $z<1.1$, depending on the EBL model used.

VERITAS is supported by grants from the U.S. Department of Energy Office of Science, the U.S. National Science Foundation and the Smithsonian Institution, and by NSERC in Canada. We acknowledge the excellent work of the technical support staff at the Fred Lawrence Whipple Observatory and at the collaborating institutions in the construction and operation of the instrument. The VERITAS Collaboration is grateful to Trevor Weekes for his seminal contributions and leadership in the field of VHE gamma-ray astrophysics, and for his interest in the wider applications of IACTs, which made this study possible.

\section{ORCID iDs}

W. Benbow (1) https://orcid.org/0000-0003-2098-170X

R. Bird (i) https://orcid.org/0000-0002-4596-8563

J. P. Finley (10) https://orcid.org/0000-0002-8925-1046

A. Furniss (i) https://orcid.org/0000-0003-1614-1273

G. H. Gillanders (10) https://orcid.org/0000-0001-8763-6252

D. Hanna (i) https://orcid.org/0000-0002-8513-5603

C. A. Johnson (i) https://orcid.org/0000-0002-0641-7320

P. Kaaret (1) https://orcid.org/0000-0002-3638-0637

D. Kieda (ㄱ) https://orcid.org/0000-0003-4785-0101

M. Krause 느 https://orcid.org/0000-0001-7595-0914

G. Maier (1) https://orcid.org/0000-0001-9868-4700

R. Mukherjee (1) https://orcid.org/0000-0002-3223-0754

D. Nieto (1) https://orcid.org/0000-0003-3343-0755

M. Pohl @ https://orcid.org/0000-0001-7861-1707

A. Popkow (i) https://orcid.org/0000-0002-3157-8839

E. Pueschel (1) https://orcid.org/0000-0002-0529-1973

G. T. Richards (1) https://orcid.org/0000-0002-1408-807X

K. Shahinyan (1) https://orcid.org/0000-0001-5128-4160

I. Telezhinsky (1) https://orcid.org/0000-0001-6678-2381

D. A. Williams (ㄷ) https://orcid.org/0000-0003-2740-9714

\section{References}

Abdo, A. A., Ackermann, M., Ajello, M., et al. 2010b, ApJS, 188, 405 Acero, F., Ackermann, M., Ajello, M., et al. 2015, ApJS, 218, 23 Ackermann, M., Ajello, M., Allafort, A., et al. 2011, ApJ, 743, 171 Ackermann, M., Ajello, M., Atwood, W. B., et al. 2016, ApJS, 222, 5 Aharonian, F., Akhperjanian, A., Barrio, J., et al. 2001, A\&A, 370, 112 Aleksić, J., Antonelli, L. A., Antoranz, P., et al. 2011, ApJL, 730, L8 Archambault, S., Archer, A., Benbow, W., et al. 2016, AJ, 151, 142

Boettcher, M., Harris, D. E., \& Krawczynski, H. 2012, in Relativistic Jets from Active Galactic Nuclei, ed. M. Boettcher, D. E. Harris, \& H. Krawczynski (Berlin: Wiley), 425

Cerruti, M., Boisson, C., \& Zech, A. 2013, A\&A, 558, A47

Cerruti, M., Zech, A., Emery, G., \& Guarin, D. 2017, in AIP Conf. Proc. 1792 , 6th Int. Symp. on High Energy Gamma-Ray Astronomy (Melville, NY: AIP), 050027

Domínguez, A., Primack, J. R., Rosario, D. J., et al. 2011, MNRAS, 410, 2556 Franceschini, A., Rodighiero, G., \& Vaccari, M. 2008, A\&A, 487, 837

Gehrels, N., Chincarini, G., Giommi, P., et al. 2004, ApJ, 611, 1005

Georganopoulos, M., Finke, J. D., \& Reyes, L. C. 2010, ApJL, 714, L157

Gilmore, R. C., Somerville, R. S., Primack, J. R., \& Domínguez, A. 2012, MNRAS, 422, 3189

Griffith, M., Langston, G., Heflin, M., et al. 1990, ApJS, 74, 129

H.E.S.S. Collaboration, Abramowski, A., Acero, F., et al. 2013, A\&A, 552, A118

Holder, J., Acciari, V. A., Aliu, E., et al. 2008, in AIP Conf. Ser. 1085, 4th Int. Meeting on High Energy Gamma-Ray Astronomy (Melville, NY), 657

Holder, J., Atkins, R. W., Badran, H. M., et al. 2006, APh, 25, 391

Kneiske, T. M., \& Dole, H. 2010, A\&A, 515, A19

Kollgaard, R. I. 1994, VA, 38, 29

Lauren-Muehleisen, S. A., Kollgaard, R. I., Ciardullo, R., et al. 1998, ApJS, 118,127

Laurent-Muehleisen, S. A., Kollgaard, R. I., Feigelson, E. D., Brinkmann, W., \& Siebert, J. 1999, ApJ, 525, 127

Massaro, E., Giommi, P., Leto, C., et al. 2009, A\&A, 495, 691

Meisner, A. M., \& Romani, R. W. 2010, ApJ, 712, 14

Petrov, L. 2011, AJ, 142, 105

Rolke, W. A., López, A. M., \& Conrad, J. 2005, NIMPA, 551, 493

Sbarufatti, B., Treves, A., \& Falomo, R. 2005, ApJ, 635, 173

Scully, S. T., Malkan, M. A., \& Stecker, F. W. 2014, ApJ, 784, 138

Tavecchio, F., Maraschi, L., \& Ghisellini, G. 1998, ApJ, 509, 608 